\title{
Análise da estrutura interna da Escala de Forças de Caráter
}

\section{Analysis of the internal structure of the Character Strengths Scale}

\section{Análisis de la estructura interna de la Escala de Fuerzas de Carácter}

\author{
Ana Paula Porto Noronha ${ }^{1}$, ORCID 0000-0001-6821-0299 \\ Helder Henrique Viana Batista ${ }^{2}$, ORCID 0000-0001-5588-8682
}

\author{
${ }^{12}$ Programa de Pós Graduação Stricto Sensu em Psicologia, Universidade São Francisco. Brasil
}

Resumo: As forças de caráter podem contribuir com a minimização de sintomas de ansiedade e depressão, uma vez que potencializam o desenvolvimento do bem-estar psicológico e subjetivo. Ainda não há consenso quanto à estrutura fatorial das forças de caráter e, em razão disso, o presente estudo teve por objetivo replicar empiricamente a classificação teórica das forças de caráter por meio de análises fatoriais exploratórias. Foram participantes 1500 estudantes universitários, com faixa etária entre 16 e 64 anos $(M=2,25 ; D P=7,96)$, que responderam à Escala de Forças de Caráter. A solução fatorial mais adequada foi a de seis fatores, com variância explicada total de $44 \%$ e confiabilidades entre .88 e .93 . Os resultados encontrados são discutidos à luz da literatura.

Palavras-chave: avaliação psicológica, psicometria, emoções, satisfação com a vida, testes psicológicos

\begin{abstract}
Character strengths can contribute to the minimization of anxiety and depression symptoms and potentiate the development of psychological and subjective well-being. There is still no consensus as to the factorial structure of the character strengths. The present study aimed to empirically replicate the theoretical classification of the character strengths through exploratory factorial analyses. A total of 1500 undergraduate students, aged between 16 and 64 years old $(M=23.25 ; S D=7.96)$, responded to the Character Strengths Scale. The most appropriate factorial solution was that of six factors, with total explained variance of $44 \%$ and reliabilities between .88 and .93 . The results are discussed in the light of the literature.
\end{abstract}

Keywords: psychological evaluation, psychometrics, emotions, life satisfaction, psychological tests

Resumen: Las fuerzas de carácter pueden contribuir a la minimización de los síntomas de ansiedad y depresión ya que potencian el desarrollo del bienestar psicológico subjetivo. Aún no hay consenso en cuanto a la estrutura factorial de las fuerzas de carácter y, en razón de ello, el presente estudio tuvo el objetivo replicar empíricamente la clasificación teórica de las fuerzas de carácter por medio de análisis factoriales exploratorios. Los participantes fueron 1500 estudiantes universitarios, con edades entre 16 y 64 años $(M=23.25 ; D E=7.96)$, que respondieron a la Escala de Fuerzas de Carácter. La solución factorial más adecuada fue la de seis factores, com varianza explicada total de $44 \%$ y confiabilidades entre .88 y .93 . Los resultados encontrados se discuten a la luz de la literatura.

Palabras clave: evaluación psicológica, psicometria, emociones, satisfacción con la vida, pruebas psicológicas 
Como citar este artigo:

Noronha, A. P. P., \& Batista, H. H. V. (2020). Análise da estrutura interna da Escala de Forças de Caráter. Ciencias Psicológicas, 14(1), e-2150. doi: https://doi.org/10.22235/cp.v14i1.2150

Correspondência: Ana Paula Porto Noronha. Universidade São Francisco, Campinas-SP. Endereço: Rua Alexandre Rodrigues Barbosa, 45, Itatiba, SP - Brasil, 13251-900.E-mail: ana.noronha@usf.edu.br. Helder Henrique Viana Batista. Universidade São Francisco, Campinas-SP. Endereço: Rua José Augusto de Mattos, 475, Campinas, SP Brasil, 13060-748. E-mail: helder.hvb@gmail.com

\section{Introdução}

O estudo das forças de caráter caracteriza-se como uma alternativa àqueles tratamentos tradicionais em psicologia que enfatizavam os aspectos psicopatológicos e as características desajustadas dos indivíduos (Peterson \& Seligman, 2004; Seligman, 2009). As forças de caráter são definidas como características positivas referentes aos comportamentos, pensamentos e sentimentos humanos que contribuem para que a bondade se desenvolva e a vida das pessoas seja melhor (Park \& Peterson, 2006; Peterson \& Seligman, 2004). A maior frequência das forças possibilita a vivência de mais emoções positivas, melhores relacionamentos entre as pessoas e maior engajamento em atividades como o trabalho, os estudos, entre outros (Littman-Ovadia, Lavy, \& Boiman-Meshita, 2017; Seligman, 2009).

A importância das forças centra-se no fato de servirem como fator de proteção contra doenças mentais, de forma que as pessoas se desenvolvam de maneira mais saudável (LitmanOvadia \& Steger, 2010), minimizando os sintomas de ansiedade e depressão (Rouse et al., 2015), e aumentando o bem-estar psicológico e subjetivo (Linley, Nielsen, Wood, Gillett, \& BiswasDiener, 2010; Oliveira, Nunes, Legal, \& Noronha, 2016) e a autorregulação emocional (Noronha \& Batista, 2020). Mais recentemente, Noronha e Campos (2018) identificaram que as forças podem ser preditas por características da personalidade, como a extroversão e a socialização. Ademais, no que se referem aos estilos parentais, entendidos como um padrão comportamental de relações entre pais e filhos, as forças de caráter apresentaram maiores associações com a responsividade do que com a exigência parental (Noronha \& Batista, 2017). Por sua vez, MartínezMarti e Ruch (2016) encontraram valores preditivos das forças em relação à resiliência, otimismo, satisfação com a vida, autoestima, apoio social, afetos positivos e autoeficácia.

Peterson e Seligman (2004) organizaram a classificação denominada Values in Action (VIA) Character of Strengths, que inclui 24 forças de caráter independentes dispostas em seis virtudes, conforme apresentado na Tabela 1. O VIA foi desenvolvido com base em uma ampla pesquisa em várias culturas, sendo considerados textos legislativos, filosóficos e religiosos (Dahlsgaard, Peterson, \& Seligman, 2005) e deu origem ao instrumento VIA-Inventory Strengths, pressupondo que a estrutura de seis dimensões fosse estável ao longo do tempo e entre culturas (Brazeau, Teatero, Rawana, Brownlee, \& Blanchette, 2012; Peterson \& Seligman, 2004).

Tabela 1.

Virtudes e disposição das forças de caráter

\begin{tabular}{ll}
\hline Virtudes ( $\mathrm{N}^{\circ}$ de forças agrupadas) & Forças de cada virtude \\
\hline Sabedoria e conhecimento (5) & Criatividade, curiosidade, pensamento crítico, amor pelo \\
& aprendizado e sensatez \\
Coragem (4) & Bravura, persistência, autenticidade e vitalidade \\
Humanidade (3) & Amor, bondade e inteligência social \\
Justiça (3) & Trabalho em equipe, imparcialidade e liderança \\
Temperança (4) & Perdão, modéstia, prudência e autorregulação \\
Transcendência (5) & Apreciação do belo, gratidão, esperança, humor e espiritualidade \\
\hline
\end{tabular}


Diferentemente de Peterson e Seligman (2004), alguns autores acreditam que as forças possuem certa interdependência entre si (Allan, 2014; Fowers, 2008; Schwartz \& Sharpe, 2006). A este respeito, cabe ressaltar que a estrutura com seis virtudes e 24 forças proposta por Peterson e Seligman (2004) não tem sido replicada empiricamente (Litman-Ovadia \& Lavy, 2012; McGrath, 2014; Neto, Neto, \& Furnham, 2014; Ng, Cao, Marsh, Tay, \& Seligman, 2016; Noronha, Dellazana-Zanon, \& Zanon, 2015; Solano \& Cosentino, 2018). Na Tabela 2 estão dispostas algumas estruturas fatoriais identificadas em estudos empíricos. As questões culturais de cada país e os contextos de aplicação são variáveis consideradas para justificar possíveis diferenças de estruturas identificadas para as forças de caráter (Ciarrochi, Atkins, Hayes, Sahdra, \& Parker, 2016), sendo necessário desenvolver mais investigações sobre o construto.

No estudo desenvolvido por Solano e Cosentino (2018) os autores investigaram as propriedades psicométricas de uma escala de forças abreviada (IVyF abreviado) e identificaram uma estrutura de três fatores para as 24 forças. Os fatores foram denominados forças interpessoais, forças de inteligência/motivação pessoal e forças de restrição. Por sua vez, McGrath (2014) analisou uma amostra transcultural, de 16 países, a fim de identificar a invariância de medida de diferentes traduções do VIA. O autor encontrou uma estrutura de cinco fatores para o VIA, os quais foram nomeados de acordo com as forças que agruparam: interpessoais, emocionais, teologais, intelectuais e de autorregulação.

A estrutura com cinco fatores também havia sido observada na adaptação do VIA para o hebraico realizada por Litman-Ovadia e Lavy (2012). Ainda que Neto et al. (2014) tenham encontrado a solução fatorial com quatro fatores como a mais adequada para o VIA, as nomenclaturas se assemelham às encontradas por McGrath (2014) e Litman-Ovadia e Lavy (2012), sendo as forças agrupadas em interpessoais, de liderança, de temperança e intelectuais. Ademais, Ng et al. (2016) identificaram uma estrutura bifatorial para o VIA, de forma que o fator geral foi denominado positividade disposicional, enquanto os fatores específicos foram sabedoria, coragem, temperança, justiça, humanidade e transcendência.

No contexto brasileiro, Noronha e Barbosa (2016) desenvolveram a Escala de Forças de Caráter (EFC) com base nos pressupostos teóricos de Peterson e Selignan (2004), porém, o instrumento não se trata de uma adaptação do VIA. No estudo de validação da EFC, Noronha et al. (2015) encontraram uma estrutura unidimensional que explicou 32\% da variância. Os últimos autores ressaltam que as divergências quanto ao modelo teórico das forças de caráter não pode ser empecilho para os pesquisadores investigarem o construto em diferentes amostras e com diferentes análises estatísticas. Desta feita, o objetivo do presente estudo foi investigar a estrutura fatorial da Escala de Forças de Caráter (EFC; Noronha \& Barbosa, 2016), por meio de análises fatoriais exploratórias, visto que a estrutura interna da EFC não foi investigada em outros estudos. 
Tabela 2

Estruturas fatoriais e agrupamentos das forças de caráter em estudos anteriores

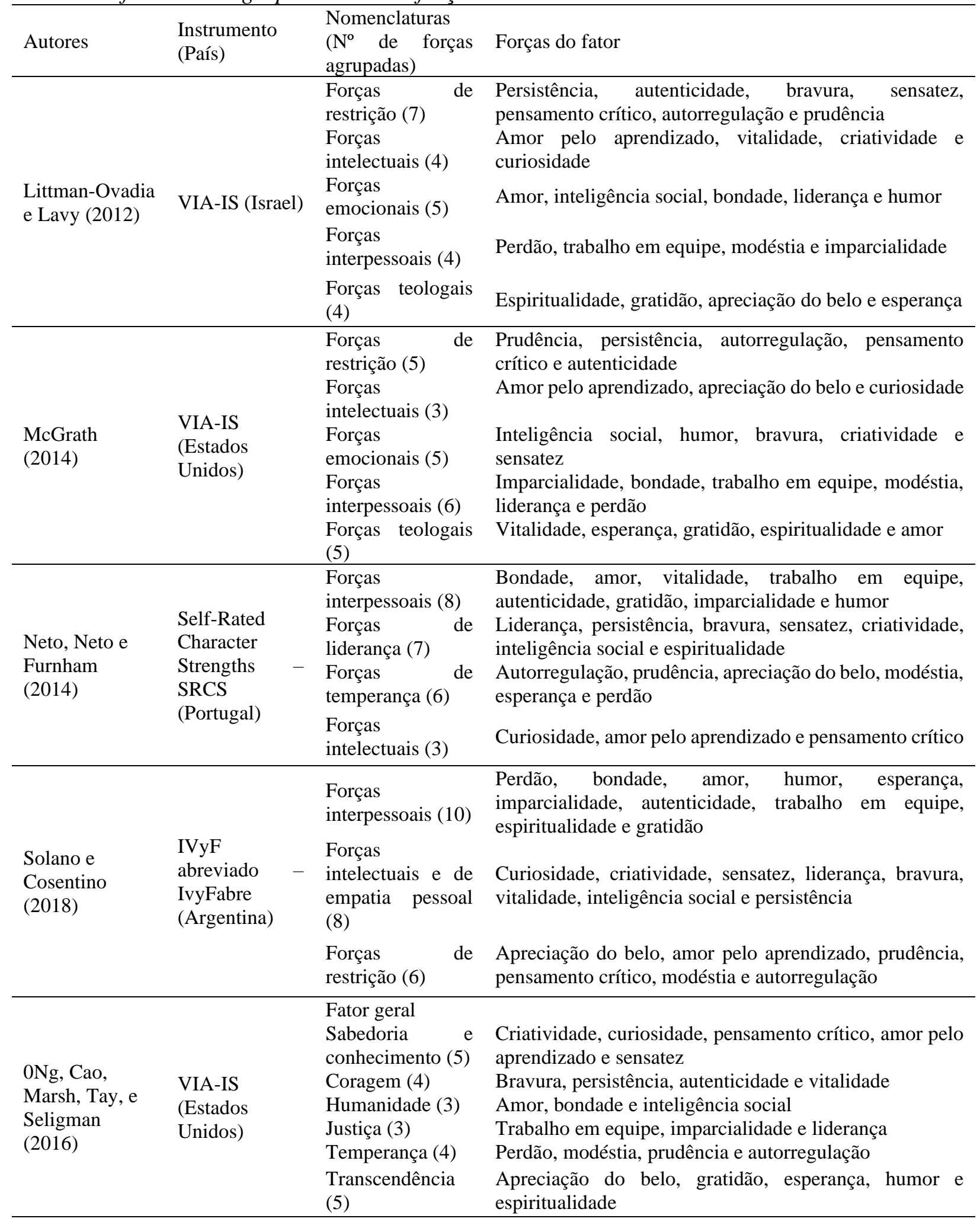




\section{Método}

\section{Participantes}

Foram participantes do presente estudo 1500 pessoas, com idades entre 16 e 64 anos $(M=$ 23,25; $D P=7,961)$, sendo 98,7\% ( $n=1481)$ do sexo feminino. Eles eram estudantes universitários de instituições privadas de ensino superior, das quais duas do estado de São Paulo e uma de Minas Gerais, todas particulares.

\section{Instrumento}

Escala de Forças de Caráter (EFC; Noronha \& Barbosa, 2016). Foi construída com base no Values in Action (VIA) Classification of Strengths (Peterson \& Seligman, 2004). Foram elaborados três itens para cada uma das 24 forças de caráter, com exceção de Apreciação do belo, que ficou com dois itens, totalizando 71 itens. Eles estão organizados em uma escala Likert, que varia de 0 (nada a ver comigo) a 4 (tudo a ver comigo) e estrutura unidimensional ( $\alpha=, 93$; Noronha et al., 2015). Exemplos de itens: "Não guardo mágoas se alguém me maltrata" e "Penso muito antes de tomar uma decisão".

\section{Procedimentos}

Após a autorização para a coleta de dados, o projeto foi encaminhado ao Comitê de Ética em Pesquisa da xx, tendo recebido parecer favorável (CAAE: 20410713.3.0000.5514). As aplicações ocorreram nas instituições de ensino, coletivamente, após a assinatura ao Termo de Consentimento Livre e Esclarecido (TCLE), para os maiores de idade, e ao Termo de Assentimento Livre e Esclarecido para aqueles menores de idade, quando autorizados pelos pais, após assinatura do TCLE. Em média, 30 minutos foram suficientes para que os participantes respondessem ao instrumento.

\section{Análise de dados}

Para realizar as análises foi utilizado o software FACTOR - versão 10.5.03 (Lorenzo-Seva $\&$ Ferrando, 2006), para itens policóricos, variando de 0 a 4 . Foram considerados o Kaiser-MeyerOlkin (KMO) e o coeficiente de Bartlett para identificar se a matriz de dados era passível de fatoração. A análise paralela (Timmerman \& Lorenzo-Seva, 2011), o MAP (Minimum Average Partial) de Velicer (1976) e o método de Hull (Lorenzo-Seva, Timmerman, \& Kiers, 2011) foram utilizados para a retenção de fatores. Os índices de ajustes observados foram o Goodness of Fit Index (GFI) e Root Mean Square of Residuals (RMSR), além da correlação de Pearson entre os fatores. Em seguida, foram conduzidas análises fatoriais exploratórias (AFE's) usando o ULS (Unweighted Least Squares) e a rotação Promin, para ativar a simplicidade dos fatores. Foi estabelecido o valor mínimo de ,30 para as cargas fatoriais. Os itens de uma mesma força que carregassem com valores acima de ,30 em mais de um fator, foram agrupados de acordo a quantidade de itens no fator, a pertinência teórica e o valor da carga fatorial. 


\section{Resultados}

Os endossos aos itens variaram de 1,77 (item 26 - Não guardo mágoas se alguém me maltrata) a 3,44 (item 50 - Acho que é importante ajudar os outros). Os desvios-padrões variaram de 0,76 (item 50) a 1,39, para o item "sou paciente" (item 12). A possibilidade de fatorialidade da matriz de dados foi confirmada pelo KMO, cujo coeficiente foi 0,93 , considerado muito bom. $\mathrm{O}$ teste de esfericidade de Bartlett's foi estimado em 44058,6 ( $\mathrm{df}=2485 ; p<, 001)$.

A AFE com método de extração de ULS e rotação Promin, escolhida para não delimitar, a priori, a interação entre os fatores, revelou uma solução fatorial inicial de 15 fatores com autovalores $>1,0$ que, juntos, explicaram $57 \%$ da variância. No entanto, a análise paralela baseada recomendou a retenção de dez fatores, o que não pareceu adequado teoricamente dados os agrupamentos de itens. Tendo em vista o objetivo do estudo, foi solicitada uma estrutura de 6 fatores em vista da classificação teórica de Peterson e Seligman (2004), com a exclusão de 8 itens que não atingiram a carga fatorial mínima de $0,30(3,26,31,36,39,55,57,59)$. A variância explicada foi de $44,23 \%$, sendo o fator 1 responsável por $22,48 \%$; o fator 2 por $5,40 \%$; o fator 3 por $5,05 \%$, fator 4 por $4,42 \%$; o fator 5 por $3,84 \%$ e o 6 por $3,04 \%$.

Quanto aos índices de ajuste, o RMSR, medida descritiva da magnitude média das correlações residuais, foi de 0,05 , portanto, aceitável para o modelo de 6 fatores. Por sua vez, o GFI foi de 0,97 , considerado ótimo ajuste. A precisão foi estimada por meio do coeficiente alfa, com base em correlações policóricas, sendo .89 para o fator 1 (12 itens); .88 para o fator 2 (12 itens); .93 para o fator 3 (12 itens); .91 para o fator 4 (14 itens); .83 para o fator 5 (7 itens) e .88 para o fator 6 (6 itens). $\mathrm{O}$ fator 1 agrupou forças que se referem ao relacionamento com o outro e foi denominado "interpessoais". O fator 2 foi nomeado "coragem", uma vez que reuniu forças de enfrentamento. O fator 3 agrupou forças de transcendência e recebeu o nome de "teologais". O fator 4 foi denominado "humanidade" por agrupar forças que indicam relacionamentos igualitários e generosos. O fator 5 recebeu o nome de "autorregulação", uma vez que reuniu forças de autocontrole. Por fim, o fator 6 recebeu o nome de intelectuais por reunir forças de aprendizado. Por fim, quanto às correlações entre os fatores, a Tabela 3 apresenta as informações. As correlações e todos os demais parâmetros são derivados da matriz de correlações policóricas, mas que são, na solução final, correlações bivariadas entre os fatores.

Tabela 3.

Coeficientes de correlação de Pearson entre os fatores da EFC - FACTOR

\begin{tabular}{llllll}
\hline Fatores & Interpessoais & Coragem & Teologais & Humanidade & Autorregulação \\
\hline Coragem & .28 & & & & \\
Teologais & .47 & .32 & & & \\
Humanidade & .43 & .44 & .47 & & \\
Autorregulação & .14 & .14 & .22 & .23 & \\
Intelectuais & .22 & .36 & .34 & .24 & -.01 \\
\hline
\end{tabular}

Os coeficientes moderados foram encontrados entre os fatores 3 e $1 ; 4$ e $1 ; 4$ e 2 e 4 e 3 . A Tabela 4 informa a estrutura fatorial e as respectivas cargas. 
Tabela 4.

Cargas fatoriais (>0.30) e Comunalidades $\left(h^{2}\right)$ da Escala de Forças de Caráter (Loading Matrix) - FACTOR

\begin{tabular}{|c|c|c|c|c|c|c|c|}
\hline Itens & $\mathrm{F} 1$ & $\mathrm{~F} 2$ & F3 & $\mathrm{F} 4$ & F5 & F6 & $\mathrm{h}^{2}$ \\
\hline 1 & .56 & .28 & .02 & -.22 & .15 & -.12 & -.12 \\
\hline 2 & .15 & -.01 & -.13 & .41 & .26 & .04 & .04 \\
\hline 4 & .37 & $.57^{a}$ & .01 & -.18 & -.03 & -.25 & -.25 \\
\hline 5 & -.16 & .06 & -.06 & .27 & .19 & .59 & .59 \\
\hline 6 & .10 & .51 & -.09 & -.07 & .16 & .07 & .07 \\
\hline 7 & -.15 & .46 & .05 & .15 & -.01 & .02 & .02 \\
\hline 8 & -.04 & .33 & $.57^{\mathrm{a}}$ & -.12 & .13 & -.03 & -.03 \\
\hline 9 & -.04 & .71 & .01 & -.05 & .13 & -.05 & -.05 \\
\hline 10 & .10 & .07 & -.15 & .47 & .07 & -.01 & -.01 \\
\hline 11 & .01 & -.05 & -.01 & $.36^{a}$ & .27 & -.13 & -.13 \\
\hline 12 & -.24 & .22 & -.01 & -.05 & .71 & -.06 & -.06 \\
\hline 13 & .14 & -.06 & .46 & -.23 & $.34^{b}$ & .32 & .32 \\
\hline 14 & $.43^{b}$ & -.12 & .17 & -.33 & .44 & .33 & .33 \\
\hline 15 & .06 & -.01 & .71 & -.05 & .06 & .10 & .10 \\
\hline 16 & .15 & -.07 & .59 & -.13 & .11 & .03 & .03 \\
\hline 17 & -.09 & .01 & -.02 & .23 & .07 & .49 & .49 \\
\hline 18 & .20 & .05 & .22 & -.26 & $.35^{\mathrm{b}}$ & .38 & .38 \\
\hline 19 & .23 & .52 & .10 & -.10 & -.13 & .11 & .11 \\
\hline 20 & .44 & .05 & .23 & .08 & -.10 & -.17 & -.17 \\
\hline 21 & .30 & -.10 & -.01 & $.40^{\mathrm{a}}$ & .25 & -.04 & -.04 \\
\hline 22 & .07 & -.14 & .50 & .31 & -.01 & -.10 & -.10 \\
\hline 23 & .02 & -.19 & .09 & .30 & -.08 & .66 & .66 \\
\hline 24 & -.01 & -.11 & .72 & .09 & .11 & .03 & .03 \\
\hline 25 & -.03 & -.15 & .11 & .28 & .01 & .69 & .69 \\
\hline 27 & .05 & .07 & .69 & -.04 & .03 & .01 & .01 \\
\hline 28 & -.11 & .12 & .80 & -.05 & -.04 & -.27 & -.27 \\
\hline 29 & -.33 & .31 & .15 & $.32^{\mathrm{b}}$ & -.01 & -.05 & -.05 \\
\hline 30 & -.02 & .45 & .09 & -.05 & .10 & .21 & .21 \\
\hline 32 & .22 & -.03 & .02 & .02 & .60 & -.12 & -.12 \\
\hline 33 &. $\mathbf{3 3}^{\mathrm{a}}$ & .09 & -.06 & .46 & -.07 & -.09 & -.09 \\
\hline 34 & .42 & .18 & .05 & .07 & .07 & .06 & .06 \\
\hline 35 & .24 & .43 & -.03 & .09 & .01 & -.04 & -.04 \\
\hline 37 & .49 & -.16 & .27 & .19 & -.05 & -.25 & -.25 \\
\hline 38 & -.26 & .42 & -.12 & -.21 & $.61^{\mathrm{a}, \mathrm{b}}$ & .06 & .06 \\
\hline 40 & -.08 & .23 & .37 & .17 & -.12 & .29 & .29 \\
\hline 41 & .46 & -.11 & .15 & .15 & -.25 & -.07 & -.07 \\
\hline 42 & .84 & -.02 & -.12 & -.16 & .03 & .08 & .08 \\
\hline 43 & .44 & .11 & -.01 & .03 & -.05 & .11 & .11 \\
\hline 44 & -.04 & -.04 & .77 & .18 & .02 & -.17 & -.17 \\
\hline 45 & -.10 & .05 & .19 & .31 & -.02 & .54 & .54 \\
\hline 46 & .17 & -.20 & -.02 & .66 & .05 & -.14 & -.14 \\
\hline 47 & -.15 & .16 & .18 & .45 & -.09 & .22 & .22 \\
\hline 48 & .22 & .43 & -.09 & -.03 & -.02 & .20 & .20 \\
\hline 49 & .03 & .18 & .65 & -.08 & .07 & .01 & .01 \\
\hline 50 & .09 & .04 & .17 & .51 & .10 & -.06 & -.06 \\
\hline 51 & -.13 & .01 & .82 & .11 & -.08 & -.27 & -.27 \\
\hline 52 & .03 & .10 & .36 & .25 & -.20 & .27 & .27 \\
\hline 53 & .14 & -.04 & .65 & -.13 & .21 & .13 & .13 \\
\hline 54 & -.09 & .03 & .16 & .33 & $.44^{\mathrm{a}, \mathrm{b}}$ & -.19 & -.19 \\
\hline 56 & -.04 & -.12 & -.03 & .71 & .04 & -.17 & -.17 \\
\hline 58 & -.11 & .12 & .09 & .53 & -.05 & -.12 & -.12 \\
\hline 60 & -.08 & .36 & .11 &. $\mathbf{3 6}^{\mathrm{b}}$ & -.06 & -.23 & -.23 \\
\hline 61 & -.03 & .44 & .24 & .15 & -.04 & -.01 & -.01 \\
\hline 62 & .23 & .62 & .04 & -.09 & -.11 & .01 & .01 \\
\hline 63 & .42 & $.47^{\mathrm{a}}$ & -.14 & .06 & .06 & -.09 & -.09 \\
\hline 64 & .78 & .17 & -.10 & -.18 & .04 & .02 & .02 \\
\hline 65 & .01 & .36 & -.07 & .37 & -.07 & -.08 & -.08 \\
\hline 66 & .06 & -.01 & -.01 & .55 & .23 & -.19 & -.19 \\
\hline 67 & .06 & -.03 & -.13 & .62 & .18 & -.02 & -.02 \\
\hline 68 & .23 & .23 & -.14 & .58 & -.05 & -.19 & -.19 \\
\hline 69 & .03 & .07 & -.23 & .33 & -.05 & .62 & .62 \\
\hline 70 & .39 & .02 & .22 & -.13 & .19 & -.08 & -.08 \\
\hline 71 & .42 & .08 & -.24 & .09 & .01 & .22 & .22 \\
\hline
\end{tabular}

Nota. Os negritos indicam os fatores os quais os itens se agruparam; F1 - Forças Interpessoais; F2 - Forças de Coragem; F3 - Forças Teologais; F4 - Forças de Humanidade; F5 - Forças de Autorregulação; F6 - Forças Intelectuais; $\mathrm{h}^{2}$ - Comunalidade; $\mathrm{a}$ - Item selecionado pelo valor da carga fatorial; $\mathrm{b}$ - Item selecionado pelo aspecto teórico. 
Em 15 itens foram observadas cargas em dois fatores, sendo que a decisão foi respeitar a manutenção no fator que tivesse maior carga, após análise teórica da pertinência.

\section{Discussões}

O presente manuscrito pretendeu investigar a estrutura fatorial da Escala de Forças (Noronha \& Barbosa, 2016). A sugestão dos métodos de retenção fatorial, com dez ou 15 fatores para a EFC, não foi identificada em estudos anteriores para as forças de caráter (Litman-Ovadia \& Lavy, 2012; McGrath, 2014; Neto, Neto, \& Furnham, 2014; Ng, Cao, Marsh, Tay, \& Seligman, 2016; Noronha, Dellazana-Zanon, \& Zanon, 2015; Solano \& Cosentino, 2018). Assim, levou-se em conta a classificação proposta por Peterson e Seligman (2004), o Values in Action (VIA) Classification of Strengths, elaborada pelos autores com base em ampla revisão documental, e fruto de cinco anos de pesquisa, de uma equipe de aproximadamente 40 pesquisadores, tal como anunciado por Waters e White (2015). O VIA organiza 24 forças de caráter, definidas como características psicológicas positivas, relativamente estáveis, traduzidas em uma analogia como ingredientes psicológicos que levam as pessoas a buscar o bem para si, para os outros e para a sociedade (Park \& Peterson, 2006, 2009; Peterson \& Seligman, 2004). O instrumento utilizado no presente estudo (EFC) foi construído com base no VIA (Noronha \& Barbosa, 2016) e teve sua estrutura interna estudada inicialmente por Noronha et al. (2015). A investigação, com análises de segunda ordem, resultou em um melhor ajuste para a solução de um fator, não confirmando a estrutura teórica de Peterson e Seligman (2004), com seis virtudes e 24 forças.

Anteriormente foram realizados estudos de validade com base na relação com outras variáveis, como personalidade e estilos parentais (Noronha \& Batista, 2017; Noronha \& Campos, 2018). Os traços de personalidade Extroversão e Socialização foram os que mais predisseram as forças de caráter (Noronha \& Campos, 2018). Em relação aos estilos parentais, as forças se correlacionaram com maiores magnitudes com a responsividade, que por sua vez, traduz o afeto, envolve sensibilidade, aceitação e compromisso (Noronha \& Batista, 2017).

Quanto aos achados do presente estudo, a versão final constou de 63 itens, sendo que o fator 1 reuniu os itens das forças Humor, Amor, Inteligência Social, Autenticidade e Apreciação do belo. $\mathrm{O}$ fator 2 incluiu as forças Sensatez, Abertura, Liderança, Trabalho em equipe, Prudência, Bravura e Criatividade. As forças Espiritualidade, Gratidão, Persistência e Otimismo se organizaram no terceiro fator, enquanto no quarto, ficaram Imparcialidade, Bondade e Modéstia. No fator 5 agruparam-se Vitalidade, Autorregulação e Perdão e, por fim no último fator, Amor pela Aprendizagem e Curiosidade. A fim de facilitar a visualização dos resultados, A Tabela 5 dispõe as forças por fator.

$\mathrm{Ng}$ et al. (2016) tentaram replicar a estrutura teórica do VIA, utilizando-se de um amplo banco de dados com mais de 400 mil participantes que responderam eletronicamente ao instrumento. No entanto, embora tenham chegado razoavelmente a uma estrutura semelhante, convém destacar que mais da metade dos 240 itens foi excluída, sendo que o instrumento permaneceu com 107. Além disso, foi encontrado um fator geral, no qual 30 itens carregaram mais fortemente nele do que no fator específico.

No presente estudo, o primeiro fator encontrado tem como núcleo central o relacionamento com o outro, possuindo leveza e conseguindo ter uma visão alegre da adversidade (Humor), estabelecendo relações de troca (Amor), com facilidade de interação (Inteligência Social) e assumindo responsabilidades pelos sentimentos e ações (Autenticidade). Possivelmente, o que o distancia disso é a Apreciação do Belo, no entanto, acrescenta a beleza, que pode ser encontrada no cotidiano. Solano e Cosentino (2018) também identificaram que as forças Humor, Amor e Autenticiadade se agruparam no mesmo fator, denominado de forças interpessoais. 
Tabela 5.

Forças de caráter distribuídas por fator

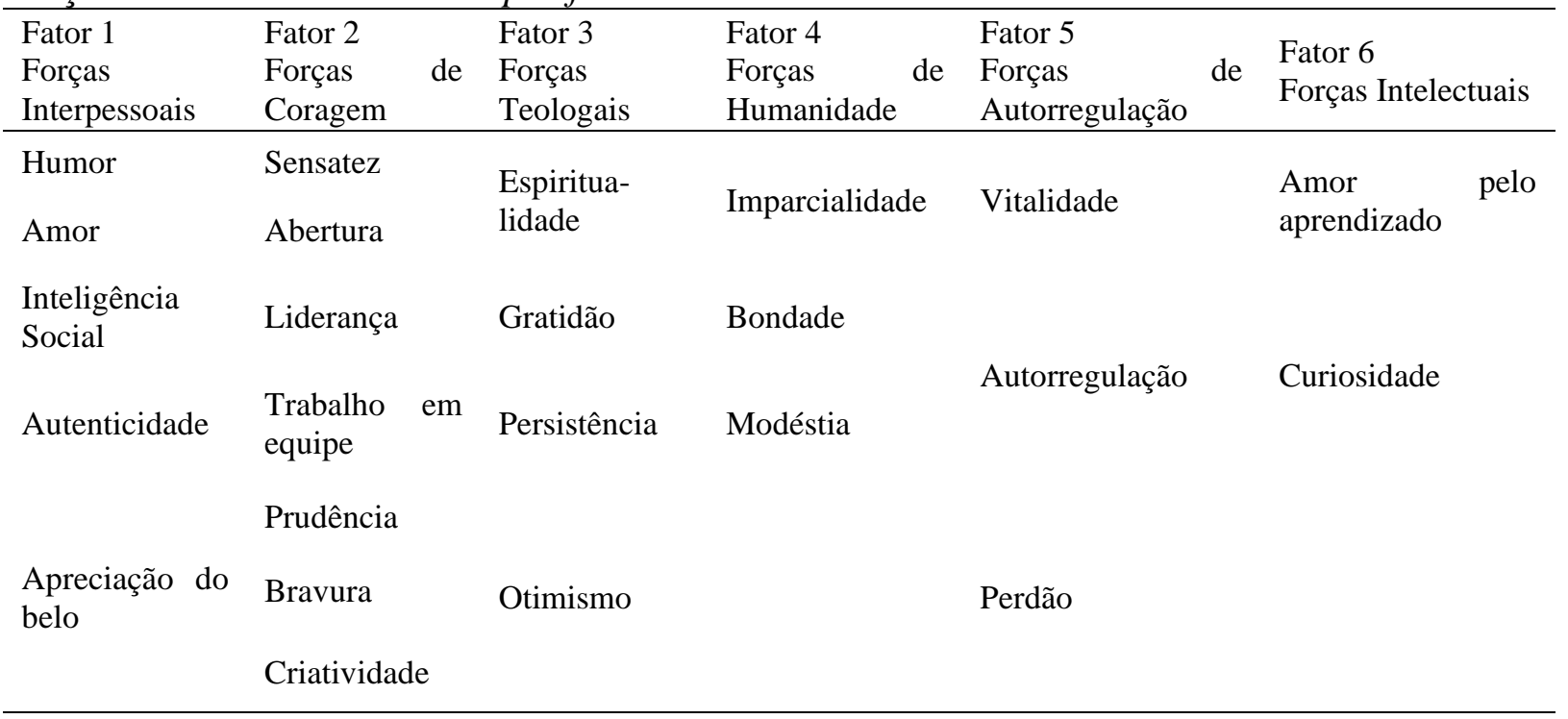

O fator 2 versa sobre características de enfrentamento, decisão ou ainda a busca de metas. Há um bloco referente à relação com o outro, como o fornecer conselhos sábios (Sensatez), trabalhar em grupo (Senso de coletividade) e incentivar um grupo (Liderança) e outro referente a um movimento do próprio indivíduo, como permitir a mudança de ideia (Pensamento crítico) e o pensamento novo e produtivo (Criatividade), refletir sobre suas escolhas (Prudência) ou ainda, não ter medo de desafios (Bravura).

O terceiro fator foi o que melhor replicou o modelo de Peterson e Seligman (2004), por incluir três forças das cinco previstas teoricamente da virtude transcendência, que diz respeito às conexões com o universo e à atribuição de significado. A perseverança, originalmente uma força da virtude coragem, poderia contribuir neste bloco com a convicção de que a persistência deve estar presente diante das dificuldades. A este respeito, há pertinência quando se toma como referência o otimismo, uma vez que as metas e as expectativas o compõem. Resultados semelhantes foram encontrados por Littman-Ovadia e Lavy (2012), exceto pela não inclusão de Perseverança.

$\mathrm{O}$ fator 4 pode ser chamado de Humanidade, especialmente pela presença da Bondade e da Modéstia, embora na virtude preconizada pelo VIA apenas a Bondade faça parte (Peterson \& Seligman, 2004). Aliado a isso está a imparcialidade, que revela o tratamento igualitário e o senso de equidade e de justiça. O fator intitulado de Interpessoal por McGrath (2014) arrolou as mesmas três forças desse estudo, acrescido de Perdão e Liderança.

As três forças que formam o fator 5 mostram coerência. Perdoar os outros e não ser vingativo (Perdão) e ter controle sobre suas emoções (Autorregulação) compõem a virtude temperança, que preza pelo controle dos excessos. Em outra medida, a Vitalidade se refere à energia vital e tem sido um importante marcador da saúde mental por suas próximas relações negativas com depressão (Rouse et al., 2015). Também a Autorregulação (AR), tal como destacado por Berking, Wirtz, Svaldi, e Hofmann (2014), pode ser importante fator de proteção para a depressão, o que foi corroborado pelos achados de Weiss, Gratz, e Lavender (2015), ao versarem sobre associações significativas e baixas entre a AR, expectativa generalizada para regulação do humor negativo e dificuldade de regulação emocional e não aceitação da emoção.

Por fim, o sexto fator agregou duas forças, Amor pela aprendizagem e Curiosidade, o que está em consonância parcialmente com os achados de Neto et al. (2014), no qual nas chamadas forças intelectuais ficaram reunidas, além da Curiosidade e do Amor pela Aprendizagem, o 
Pensamento Crítico. De modo semelhante, nos achados de McGrath (2014), a terceira força a compor o fator foi Apreciação pelo Belo, e não Pensamento Crítico.

Possivelmente, as diferenças encontradas entre o modelo teórico de Peterson e Seligman (2004) e os resultados do presente estudo, se explicam pelo fato de existir certa interdependência entre as forças (Fowers, 2008; Schatz \& Sharpe, 2006). Allan (2014) considerou a importância de investigar as forças considerando alguns pares (por exemplo, honestidade e bondade; amor e inteligência social), uma vez que, isoladamente as forças podem ser menos efetivas do que se utilizadas em conjunto. As forças intelectuais encontradas no fator 6 aproximam-se da proposta de Allan (2014), à medida que a Curiosidade indica interesse, procura de novidades, sede de saber e o Amor pelo aprendizado diz respeito ao conhecimento mais sistematizado, mesmo sem incentivos exteriores. Ou seja, ter Curiosidade sem o Amor pelo aprendizado pode levar o indivíduo a somente se interessar por algum tema, mas sem buscar um aprofundamento e, consequentemente, crescimento em determinados assuntos.

\section{Conclusões}

Os resultados da presente investigação caminham no mesmo sentido de outros estudos, indicando que o modelo teórico proposto na elaboração do VIA por Peterson e Seligan (2004) não tem sustentação empírica. Ainda que tenha sido identificada uma estrutura com seis fatores, as forças de caráter se agruparam de outra maneira nos fatores quando comparadas com a proposição teórica original.

Como limitações do presente estudo, considera-se o fato de não ter sido realizadas análises que pudessem indicar que as características sociodemográficas dos participantes interferiam na forma de responder aos itens, o que minimizaria alguns possíveis vieses da escala. Nesse sentido, como agenda de estudos futuros, faz-se importante considerar análises que investiguem o funcionamento diferencial, aquiescência, desejabilidade social e o poder discriminativo dos itens a fim de compreender melhor tais disposições fatoriais.

\section{Referências}

Allan, B. A. (2014). Balance among character strengths and meaning in life. Journal of Happiness Studies, 16(5), 1247-1261. doi: 10.1007/s10902-014-9557-9

Berking, M., Wirtz, C. M., Svaldi, J., \& Hofmann, S. G. (2014). Emotion Regulation Predicts Symptoms of Depression over Five Years. Behavior Research and Therapy, 57(1), 13-20. doi: $10.1016 /$ j.brat.2014.03.003

Brazeau, J. N., Teatero, M. L., Rawana, E. P., Brownlee, K., \& Blanchette, L. R. (2012). The Strengths Assessment Inventory: of a New Measure of Psychosocial Strengths for Youth. Journal of Child and Family Studies, 21(3), 384-390. doi: 10.1007/s10826-011-9489-5

Ciarrochi, J., Atkins, P. W. B., Hayes, L. L., Sahdra, B. K., \& Parker, P. (2016). Contextual positive psychology: Policy recommendations for implementing positive psychology into schools. Frontiers in Psychology, 7(1561), 1-16. doi: 10.3389/fpsyg.2016.01561.

Dahlsgaard, K., Peterson, C., \& Seligman, M. E. P. (2005). Shared virtue: The convergence of valued human strengths across culture and history. Review of General Psychology, 9(3), 203-213. doi: 10.1037/1089-2680.9.3.203

Fowers, B. J. (2008). From continence to virtue: Recovering goodness, character unity, and character types for positive psychology. Theory \& Psychology, 18(5), 629-653. doi:10.1177/0959354308093399

Linley, P. A., Nielsen, K. M., Wood, A. M., Gillett, R., \& Biswas-Diener, R. (2010). Using Signature Strengths in Pursuit of Goals: Effects on Goal Progress, Need Satisfaction, and Well-being, and Implications for Coaching Psychologists. International Coaching 
Psychology Review, 5(1), 6-15. Recuperado de http://www.enhancingpeople.com/paginas/master/Bibliografia_MCP/Biblio05/USING\%2 OSIGNATURE\%20STRENGTHS\%20IN\%20PURSUIT\%20GOALS.pdf

Littman-Ovadia, H., \& Lavy, S. (2012). Character strengths in Israel Hebrew adaptation of the VIA Inventory of Strengths. European Journal of Psychological Assessment, 28(1), 41-50. doi: 10.1027/1015-5759/a00008

Littman-Ovadia, H., Lavy, S., \& Boiman-Meshita, M. (2017). When theory and research collide: examining correlates of signature strengths use at work. Journal of Happiness Studies, 18(2), 527-548. doi: 10.1007/s10902-016-9739-8

Littman-Ovadia, H., \& Steger, M. (2010). Character Strengths and Well-being Among Volunteers and Employees: Toward an Integrative Model. The Journal of Positive Psychology Publication, 5(6), 419-430. doi:10.1080/17439760.2010.516765

Lorenzo-Seva, U., \& Ferrando, P. J. (2006). FACTOR: A computer program to fit the exploratory factor analyses model. Behavior Research Methods, 38(1), 88-91. doi: 10.3758/BF03192753

Martínez-Martí, M. L., \& Ruch, W. (2016). Character strengths predict resilience over and above positive affect, self-efficacy, optimism, social support, self-esteem, and life satisfaction. The Journal of Positive Psychology, 12(2), 1-10. doi: 10.1080/17439760.2016.1163403

McGrath, R. E. (2014). Measurement Invariance in Translations of the VIA Inventory of Strengths. European Journal of Psychological Assessment, 32(3), 187-194. doi: 10.1027/10155759/a000248

Neto, J., Neto, F., \& Furnham, A. (2014). Gender and Psychological Correlates of Self- rated Strengths Among Youth. Social Indicators Research, 118(1), 315-327. doi: 10.1007/s11205-013-0417-5

Ng, V., Cao, M., Marsh, H. W., Tay, L., \& Seligman, M. E. P. (2016). The factor structure of the Values in Action Inventory of Strengths (VIA-IS): An item-level Exploratory Structural Equation Modeling (ESEM) bifactor analysis. Psychological Assessment, 29(8). doi: 10.1037/pas0000396

Noronha, A. P. P., \& Barbosa, A. J. G. (2016). Forças e virtudes: Escala de Forças de Caráter. In C. S. Hutz (Org.), Avaliação em Psicologia Positiva: Técnicas e Medidas (pp. 21-43). São Paulo: CETEPP.

Noronha, A. P. P., \& Batista, H. H. V. (2017). Escala de Forças e Estilos Parentais: Estudo correlacional. Estudos Interdisciplinares em Psicologia, 8(2), 2-19. doi: 10.5433/22366407.2017v8n2p02

Noronha, A. P. P., \& Batista, H. H. V. (2020). Relações entre forças de caráter e autorregulação emocional em universitários brasileiros. Revista Colombiana de Psicología, 29(1), 73-86. doi: $10.15446 / . v 29 n 1.72960$

Noronha, A. P. P., \& Campos, R. R. F. (2018). Relationship between character strengths and personality traits. Estudos de Psicologia, 35(1), 29-37. doi: 10.1590/198202752018000100004

Noronha, A. P. P., Dellazzana-Zanon, L. L., \& Zanon, C. (2015). Internal structure of the Characters Strengths Scale in Brazil. Psico-USF, 20(2), 229-235. doi: 10.1590/141382712015200204

Oliveira, C., Nunes, M. F. O., Legal, E. J., \& Noronha, A. P. P. (2016). Bem-Estar Subjetivo: estudo de correlação com as Forças de Caráter. Avaliação Psicológica, 15(2), 177-185. doi: 10.15689/ap.2016.1502.06

Park, N., \& Peterson, C. (2006). Moral competence and character strengths among adolescents: The development and validation of the Values in Action Inventory of Strengths for Youth. Journal of Adolescence, 29(6), 891-909. doi: 10.1016/j.adolescence.2006.04.011 
Park, N., \& Peterson, P. (2009). Character strengths: Research and practice. Journal of College \& Character, 10(4). doi: 10.2202/1940-1639.1042

Peterson, C. E., \& Seligman, M. E. P. (2004). Character strengths and virtues. New York, NY: Oxford University Press.

Rouse, P. C., Jet, J. J. C. S., Zanten, V. V., Ntoumanis, N., Metsios, G. S., Yu, C., ..., \& Duda, J. L. (2015). Measuring the positive psychological well- being of people with rheumatoid arthritis: A cross-sectional validation of the subjective vitality scale. Arthritis Research \& Therapy, 17(1), 1-7. doi: 10.1186/s13075-015-0827-7

Schwartz, B., \& Sharpe, K. E. (2006). Practical wisdom: Aristotle meets positive psychology. Journal of Happiness Studies, 7(3), 377-395. doi: 10.1007/s10902-005-3651-y.

Seligman, M. E. P. (2009). Felicidade autêntica: usando a Psicologia Positiva para a realização permanente. Rio de Janeiro: Objetiva.

Solano, A. C., \& Cosentino, A. C. (2018). IVyF abreviado - IVyFabre-: análisis psicométrico y de estructura factorial en Argentina. Avances en Psicología Latinoamericana/Bogotá (Colombia), 36(3), 619-637. doi: 10.12804/revistas.urosario.edu.co/apl/a.4681

Timmerman, M. E., \& Lorenzo-Seva, U. (2011). Dimensionality assessment of ordered polytomous items with parallel analysis. Psychological Methods, 16(2), 209-220. doi: $10.1037 / \mathrm{a} 0023353$

Waters, L., \& White, M. (2015). Case study of a school wellbeing initiative: Using appreciative inquiry to support positive change. International Journal of Wellbeing, 5(1), 19-32. doi: 10.5502/ijw.v5i1.2

Weiss, N. H., Gratz, K. L., \& Lavender, J. M. (2015). Factor structure and initial validation of a multidimensional measure of difficulties in the regulation of positive emotions: The DERSPositive. Behavior Modification, 39(3), 431-453. doi: 10.1177/0145445514566504

Financiamento: O presente trabalho foi realizado com apoio da Coordenação de Aperfeiçoamento de Pessoal e Nível Superior - Brasil (CAPES) - Código de Financiamento 001

Participação dos autores: a) Planejamento e concepção do trabalho; b) Coleta de dados; c) Análise e interpretação de dados; d) Redação do manuscrito; e) Revisão crítica do manuscrito.

A.P.P.N. contribuiu em a, b, c, d, e; H.H.V.B. em c, d, e.

Editora científica responsável: Dra. Cecilia Cracco 